\title{
IMPLEMENTACIÓN DE UNA UNIDAD REGULADORA DE TENSIÓN CC DE POTENCIA DE BAJO COSTO BASADA EN MICROCONTROLADOR
}

\section{IMPlEMENTATion of a Low-Cost Voltage Drop Diode Unit Based on Micro-Controller}

\author{
Rene A. Capitanio, Ruben B. Godoy, José Montalván B., Danucha M. Marchesin y \\ JoÁo O. P. Pinto
}

Laboratory of Artificial Intelligence, Power Electronic, Digital Electronic and Electrical Drives BATLAB - Federal University of Mato Grosso do Sul - Brasil

\section{RESUMEN}

El presente trabajo busca el desarrollo de una salida de tensión regulada DC con la topologia llamada Unidad de Diodos de Caída de Voltaje (UDCV). El sistema está compuesto por dos partes: el circuito de potencia y el circuito de procesamiento de señales. La placa de potencia contiene los circuitos responsables para alimentar el contactor y la placa de procesamiento de señales. Las señales acondicionadas son procesadas en el microcontrolador. El objetivo del sistema es mantener el voltaje para $125 \mathrm{VCC} \pm 4 \%$, para una potencia de salida de $25 \mathrm{~kW}$. Por consiguiente, alimenta un sistema de telecomunicaciones PBAX que está aislado de la red eléctrica de distribución con un banco de baterias. El firmware fue desarrollado en el ambiente MPLAB para el microcontrolador 18F6620. Los resultados obtenidos muestran que el sistema propuesto es satisfactorio y que también representa un costo más bajo de implementación que las topologías existentes.

Descriptores: Unidad de Diodos de Caida de Voltaje (UDCV), regulación de tensión CC, electrónica de potencia, sistemas integrados, circuitos y sistemas

\begin{abstract}
The present work aims to develop a DC voltage output regulated with a topology called Voltage Drop Diode Unit (VDDU). The system is composed of two parts: a power circuit, and a signal processing circuit. The power board contains the responsible circuits to supply the contactor and the signal processing control board. The conditioned signals are processed in a micro-controller. The objective of the system is to maintain the voltage supply around 125 $V D C+/-4 \%$, for a power of $25 k W$. Thereby supplying a PBAX telecommunication system that is isolated of the electrical network distribution with a battery array. The firmware was developed in the MPLAB environment for the micro-controller 18F6620. The obtained results show that the proposed system is satisfactory and that it also presents a lower economic cost of implementation than the existent converter topologies.
\end{abstract}

Keywords: Voltage Drop Diode Unit (VDDU), DC voltage regulation, power electronics, embedded system, circuits and systems 


\section{INTRODUCCIÓN}

En [1] se describe una topología compuesta por ocho transistores en paralelo con diodos que ofrecen una regulación VDC para una potencia de $1 \mathrm{~kW}$. En [2] se presenta la topologia multifase Buck (ocho transistores y tres inductores) con un control basado en FPGA como una regulación de tensión de +- $0,5 \%$. Sin embargo, ofrece un pequeño rango de salida de voltaje CC para una corriente de 40A.

Para los convertidores desarrollados en [3] y [4], se usó la topologia usada en el circuito Boost en paralelo con un rectificador de onda completa. Los principales objetivos del sistema son: alto factor de potencia, baja distorsión armónica (TDH) de corriente y alta eficiencia. Sin embargo, el sistema no ofrece regulación en la salida de voltaje.

En [5], [6] y [7] se presentan las desventajas de las topologias de los convertidores CC-CC. Estos convertidores necesitan grandes inductores, transistores de potencia o MOSFET, y grandes capacitores para el nivel de corriente deseado. En [8] y [9] se observa que la regulación lineal no presenta una buena eficiencia.

La principal función UDCV es regular la salida de tensión DC de la fuente, y añadir o quitar los diodos de potencia. Para tal fin, se usan contactores en paralelos, los cuales son activados por una señal que proviene de un microcontrolador.

\section{Modelo matemático}

El voltaje de caída depende de la corriente de carga, como se muestra en la figura 1. Este trabajo aproxima una función cuadrática a una función lineal entre la región de mínima y máxima corriente.

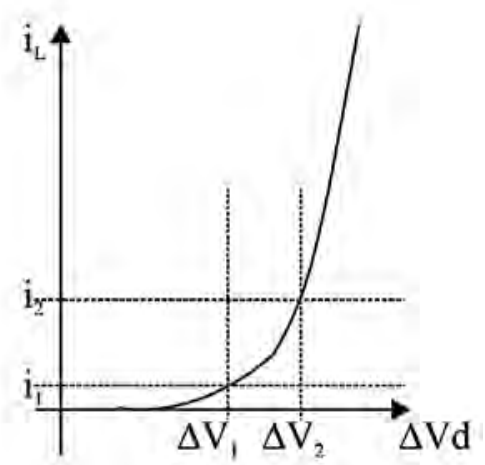

Figura 1: Caida de tensión del diodo.

$$
\begin{aligned}
& V_{o}(t)=V_{i}(t)-n_{x} \times \Delta V_{d n}\left(i_{\text {load }}\right) \\
& V_{i_{1}}<V_{i}(t)<V_{i_{2}} \\
& V o_{1}<V_{o}(t)<V o_{2} \\
& \Delta V_{i_{-} \text {load_min }}<\Delta V_{\text {dn }}\left(i_{\text {load }}\right)<\Delta V_{i_{-} \text {load_max }}
\end{aligned}
$$

donde:

$n_{x} \quad$ : número de unidades de caída de tensión activadas por una señal lógica 0 o 1 ,

$V_{i}(t) \quad:$ tensión de entrada,

$V_{o}(t) \quad:$ tensión de salida, $\mathrm{y}$

$\Delta V_{d n}\left(i_{\text {load }}\right)$ : voltaje de salida variable con la corriente de la carga.

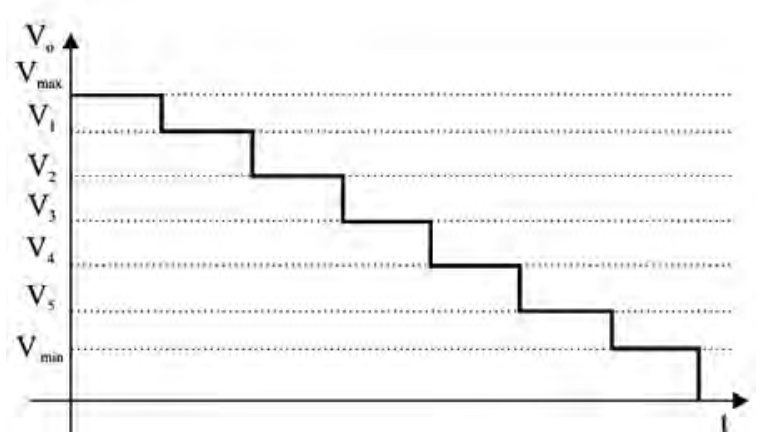

Figura 2: Caida de tensión para el retificador de configurado en cinco niveles.

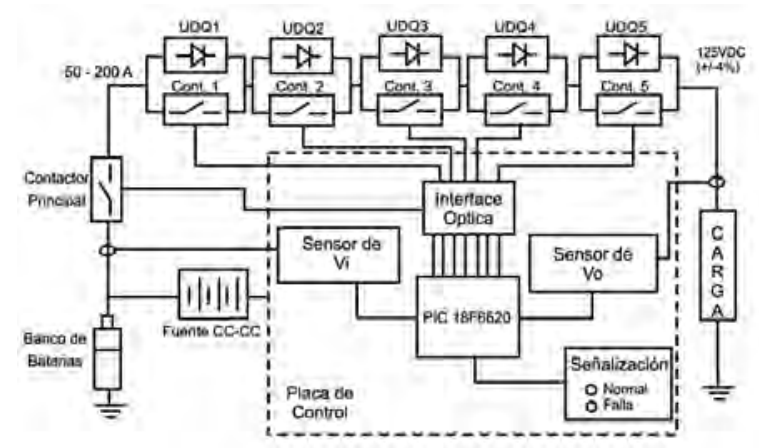

Figura 3: Muestra el UDCV bloque general.

\section{Control propuesto}

El UDCV no usa técnicas complejas de control, como los convertidores CC-CC o los rectificadores monofásicos controlados usualmente encontrados.

El control de la planta está basado en un algoritmo recurrente que mide constantemente $\mathrm{Vi}$ y Vo. Si Vo es mayor que 130VCC, el control insertará una unidad de caída de tensión y volverá a medir la tensión de salida. Este es un proceso iterativo 
que se dará hasta que la tensión quede en el rango solicitado.

Si todas las unidades de caída de tensión están actualmente conduciendo y Vo permanece por encima de 130VCC, el sistema de protección desconectará el rectificador con el contactor principal.

Si la Vi es menor de 120VCC y todas las unidades están cortocircuitadas, el sistema envía una señal para abrir el contactor principal y asegurar la protección de la batería.

\section{Descripción del sistema propuesto}

Para el sistema desarrollado se consideró algunas normas consultadas en [10], [11] y [12]. La figura 3 muestra el diagrama de bloques general diseñado para el UCDV.

El diodo seleccionado para la aplicación fue el SKN 240, como se muestra en la sección II. La tensión de caída depende de la corriente de carga. Siguiendo la ecuación (5) para 50A, la caída de tensión será de 2,8VCC para cada nivel de configuración. De esta manera, para la carga de 200a, la caída de tensión será 4,6V para cada unidad de diodos.

La ecuación (5) muestra la potencia disipada para una corriente de 200A. La pérdida obtenida para $50 \mathrm{~A}$ fue de $0,7 \mathrm{~kW}$ y para $200 \mathrm{~A}$ de $4,6 \mathrm{~kW}$, lo cual resulta en una eficiencia media del $90 \%$.

$$
P_{\text {disipada }}=\Delta V_{d} \cdot \frac{\text { diodos }}{\text { nivel }} \cdot N_{\text {niveles }} I_{L}
$$

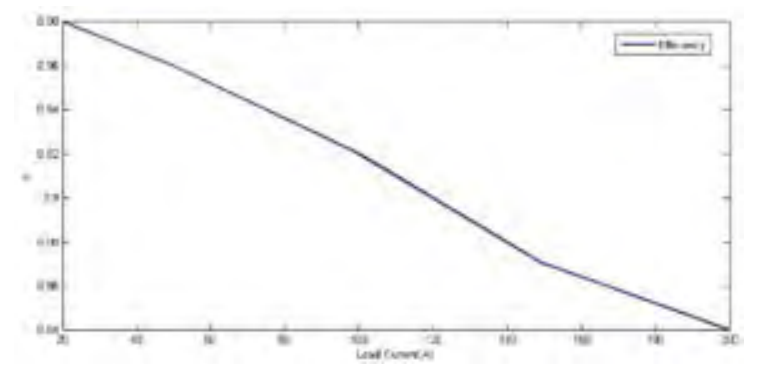

Figura 4: Eficiencia del sistema según la variación de carga.

El contactor escogido para esta aplicación fue el RMT 31031 de Emerson. El núcleo es de tipo energizado, que es el principal contactor cerrado con pulso positivo. La condición es mantenida hasta que se aplica el pulso negativo sobre la entrada, abriendo el contactor.

El contactor tiene una tensión de operación de 150 VCC y una máxima corriente de 200A. El núcleo del contactor fue alimentado con una fuente de 30VCC.

\section{Algoritmo implementado}

El algoritmo fue implementado en el micricontrolador PIC 18F6620 del fabricante Microchip. Para este microcontrolador se utilizó la interface MPLAB con el lenguaje C.

Se compuso el algoritmo general para la iniciado, evaluación y monitoreo de las rutinas. El primer paso del algoritmo es la rutina de inicio que solo incluye los comandos básicos para el inicio del microcontrolador (frecuencia, canales $\mathrm{A} / \mathrm{D}$, puertas $\mathrm{I} / \mathrm{O}$, etc.). Por esta razón, no será explicado en este artículo.

Describirenos los siguientes ítems: rutina de pruebas y rutina de monitoreo.

\section{Rutina de pruebas}

Se lleva a cabo una rutina de prueba toda vez que el sistema es iniciado. Esta rutina es responsable de la inspección de la operación correcta del sistema periférico. Se puede dividir en cinco partes (fig. 6):

1era. prueba: Alarmas y verificación de la señales. Se evalúan todos los LED del panel frontal con el objetivo de verificar su correcta operación. También chequea si las alarmas de contacto seco están reportando las fallas correctamente a la unidad central de control.

2da. prueba: Verificación de voltaje de batería. Se prueba la tensión de desconexión basada en la tensión seleccionada. Este voltaje puede asumir tres valores diferentes: 101,5105 y 108,5 VCC. Este valor seleccionado es instantáneamente comparado con la medida de entrada de tensión. Si el valor es inferior al valor seleccionado, el control de la unidad envía un comando al contactor principal para abrirlo. En este caso, se actualiza un LED en el panel frontal del UDCV y se emite una alarma a la unidad central. 
3era. prueba: Operación del contactor. Consiste en abrir y cerrar inmediatamente el contactor. Se verifican los dos estados para cerciorarse de que los dos han ocurrido correctamente. Para esto, se usa el monitoreo de una señal de los contactos auxiliares.

4ta. prueba: Caída de voltaje del diodo. Primero se abre el contactor y se mide la caída de tensión; luego se cierra y se conecta una pequeña carga al sistema, donde el valor a ser comparado es relacionado con una mínima caída del diodo.

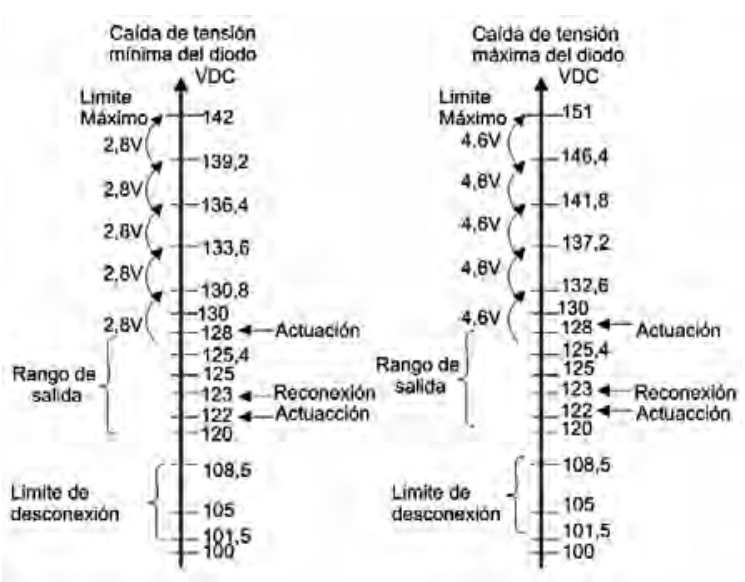

Figura 5: Variación de las tensiones de caída de UDCV.

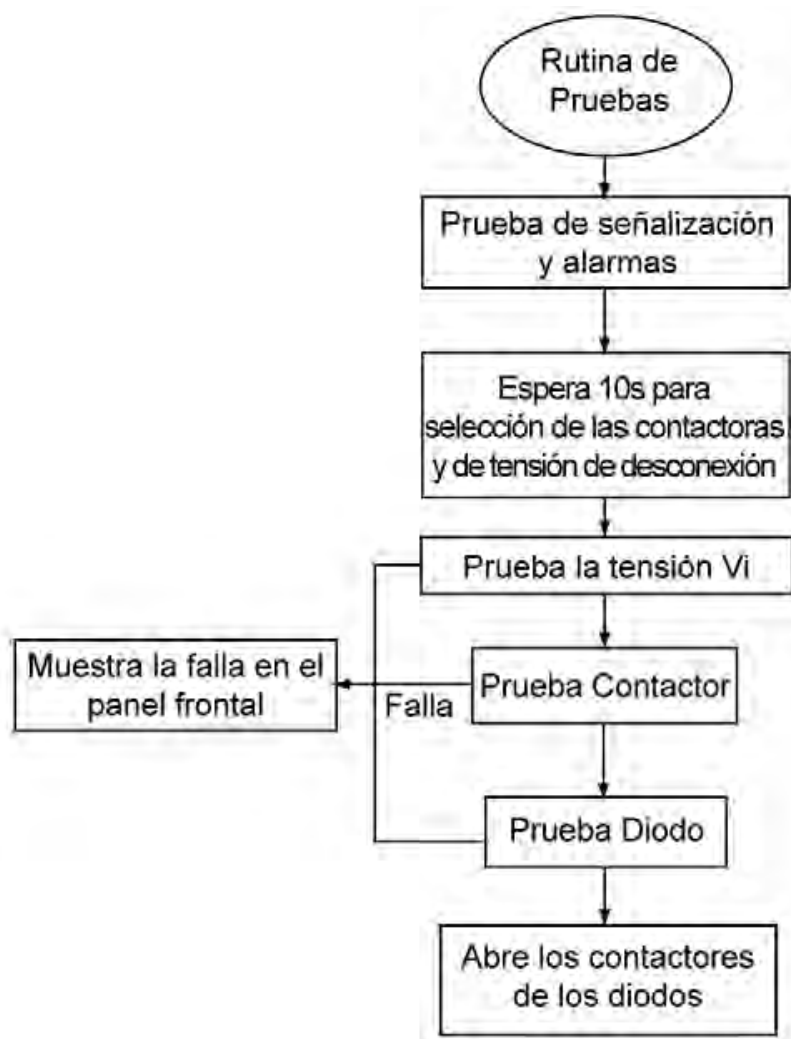

Figura 6: Diagrama de la rutina de pruebas. 5ta. prueba: Fallas. En esta primera parte de la rutina de prueba, se usan todos los contactores para verificar la presencia de fallas. Para todos los casos se verifica el funcionamiento de los respectivos dispositivos. Si ocurre alguna falla en los diodos o el contactor, esto se mostrará en el panel frontal del UDCV.

Finalizadas todas las pruebas y no se reporta ninguna falla, el procesador vuelve a la rutina principal y espera a que se dé un nuevo reinicio (botón de reset). Cuando esto ocurre, el sistema entra en las rutinas de monitoreo continuo.

\section{Rutina de monitoreo}

La rutina tiene dos pasos: En el primero, se verifica si ha ocurrido alguna falla buscando un evento en la memoria EEPROM, y se revisa el sistema para ver cuántos contactores han sido usados. En el segundo paso, se confirma si la batería presenta alguna falla. Si el voltaje es menor de 123VCC, el sistema abre el contator principal para evitar daños en la batería y enviar una señal de alarma al panel frontal y a la alarma sonora.

Terminadas las pruebas, el sistema entra en la rutina de monitoreo de lazo continuo mostrado en la figura 7 .

Tabla I: Comparación de las diferentes topologias con el UDCV.

\begin{tabular}{|c|c|c|c|}
\hline Topología & Aplicación & Desventaja & Ventaja \\
\hline Buck & $\begin{array}{c}\text { Reductor de } \\
\text { Tensión } \\
\text { DC }\end{array}$ & \multirow{2}{*}{$\begin{array}{c}\text { El inductor y } \\
\text { los transistores } \\
\text { se vuelven } \\
\text { mas caros, } \\
\text { para una alta } \\
\text { corriente de } \\
\text { carga. }\end{array}$} & \multirow{3}{*}{$\begin{array}{c}\text { Control } \\
\text { Robusto } \\
\text { en malla } \\
\text { cerrada }\end{array}$} \\
\hline $\begin{array}{l}\text { Buck- } \\
\text { Boost }\end{array}$ & $\begin{array}{c}\text { Bajador } \\
\text { Elevador } \\
\text { de Tensión }\end{array}$ & & \\
\hline $\begin{array}{c}\text { Regulador } \\
\text { Lineal }\end{array}$ & $\begin{array}{c}\text { Regulador de } \\
\text { Voltaje }\end{array}$ & \begin{tabular}{|c|} 
Baja \\
eficiencia, baja \\
corriente
\end{tabular} & \\
\hline UDCV & $\begin{array}{c}\text { Alta } \\
\text { Corriente }\end{array}$ & $\begin{array}{c}\text { Control lento, } \\
\text { no proteje } \\
\text { de picos de } \\
\text { tensión o } \\
\text { transistorios }\end{array}$ & \begin{tabular}{|c} 
Bajo \\
costo, \\
buena \\
eficiencia, \\
enfocado \\
a la \\
aplicación
\end{tabular} \\
\hline
\end{tabular}




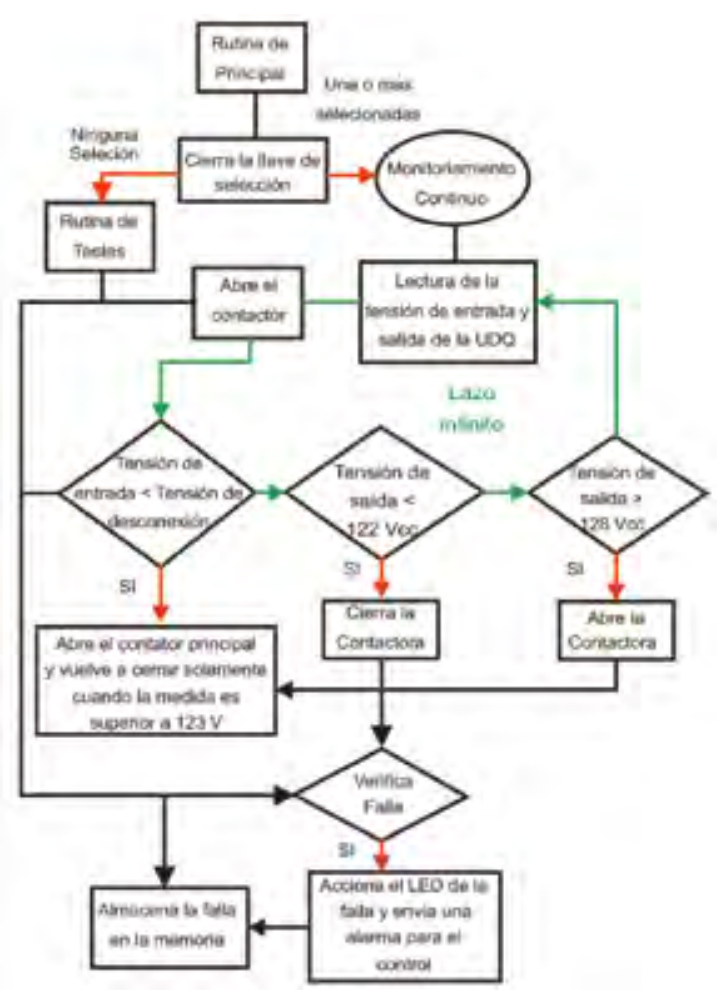

Figura 7: Diagrama de la rutina de monitoreo.

\section{Sistema implementado}

La implementación del control se divide en dos sistemas: fuentes de tensión y placa de procesamiento de señales. El conjunto de adquisición, compuesto por dos sistemas, se divide en la medición de la tensión de entrada y la medición de la tensión de salida, así como el acondicionamiento de señales del microcontrolador. La descripción de los circuitos se realiza en la secuencia.

Se usa una fuente de conmutación tipo aislada Flyback para la aplicación descrita en [20]. Se simula una fuente flyback controlada por un control PWM basado en el CI UC3845 de 30VCC. Se la requirió para alimentar el núcleo de los contactores. Hay otras dos fuentes de tensión flyback que forman dos bloques idénticos con salidas de 5VCC, 15VCC y -15VCC, las cuales alimentan separadamente la parte de potencia y la parte control. También se desarrollan filtros de EMI para la aplicación.

Para la implementación de la placa de control se requieren: acopladores ópticos (TLP621) en la entrada del microcontrolador, amplificadores (ISO 124), rectificadores de onda completa (L298) para la actuación del contactor, relays de contactos secos, transistores (BC548), resistores y diodos LEDs para la señalización.

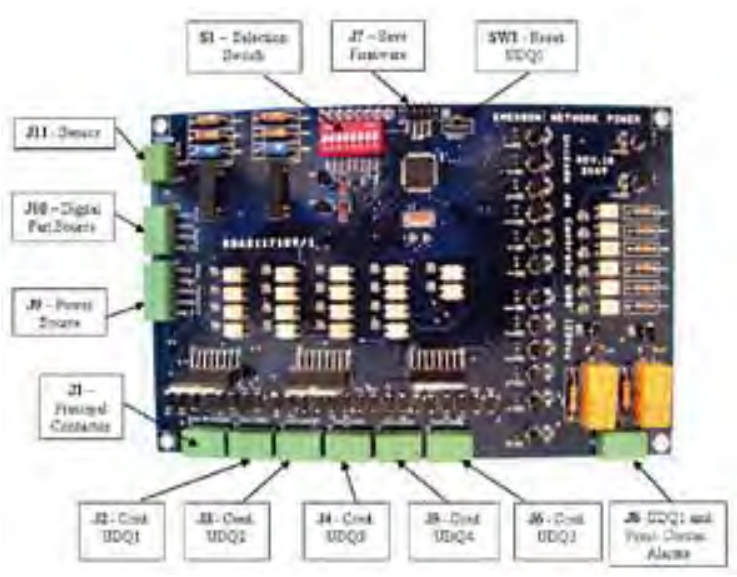

Figura 8: Unidad de control de UDCV.

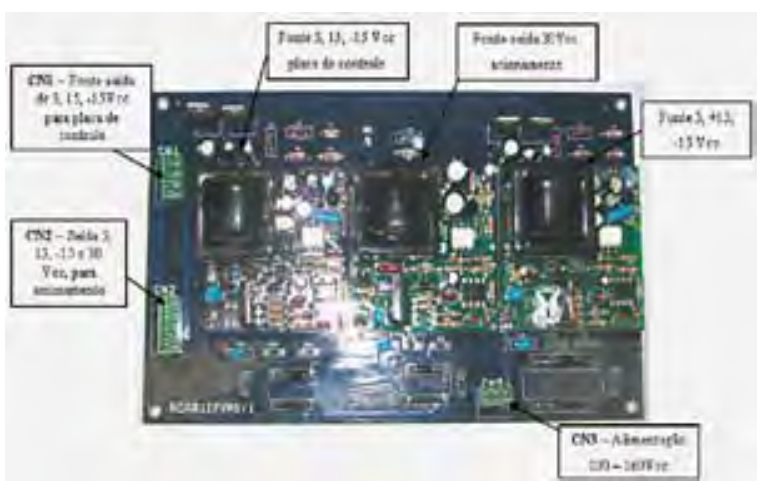

Figura 9: Fuentes de tensión implementadas.

\section{RESULTADOS}

Los resultados obtenidos para el UDCV se muestran en las figuras 10, 11, 12 y 13. En todos los gráficos se presentan sin carga en un prototipo de baja potencia usado en el laboratorio.

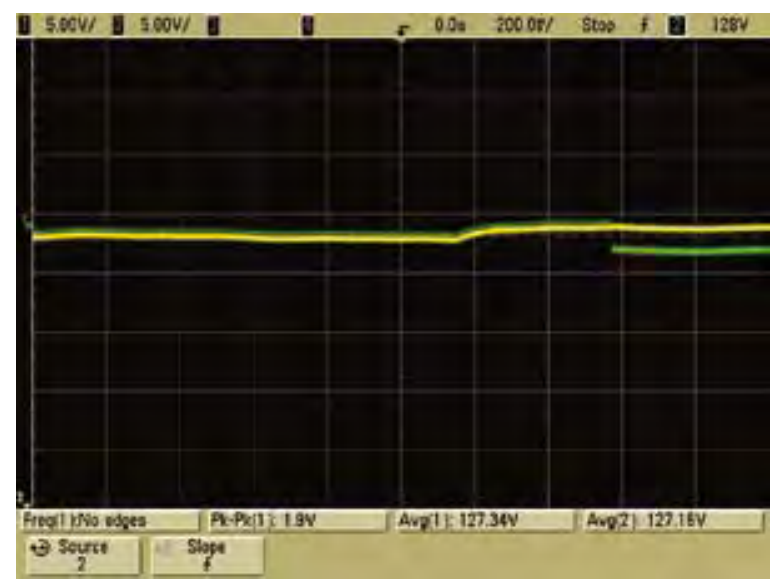

Figura 10: Primera caída de tensión para asegurar la salida en $128 \mathrm{VCC}$. 
Las figuras 10 y 11 representan la inserción de las unidades de caída bajo el límite de 128VCC. Las figuras 12 y 13 representan las unidades de caída removidas cuando la salida de tensión alcanza el mínimo de 122VCC. Los límites 122 VCC y 128VCC son representados por la red punteada en cada figura. La línea amarilla es la tensión de entrada y la línea verde la tensión de salida.

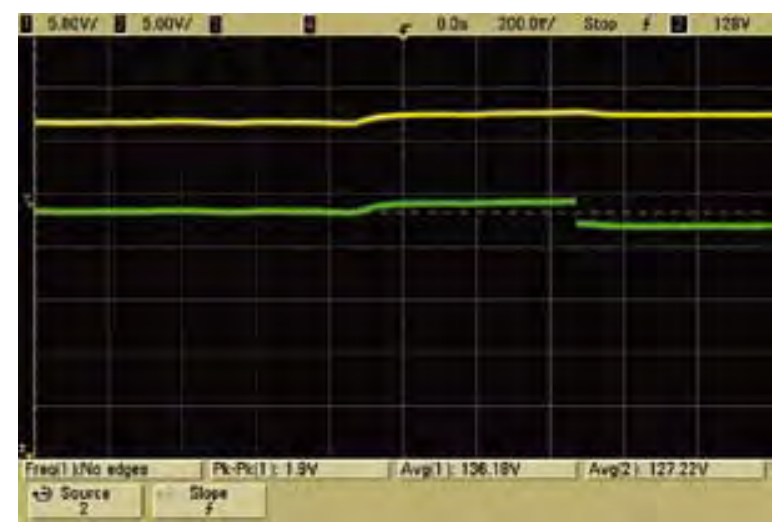

Figura 11: Quinta caida de tensión con una entrada de 136VCC y limite en 128VCC.

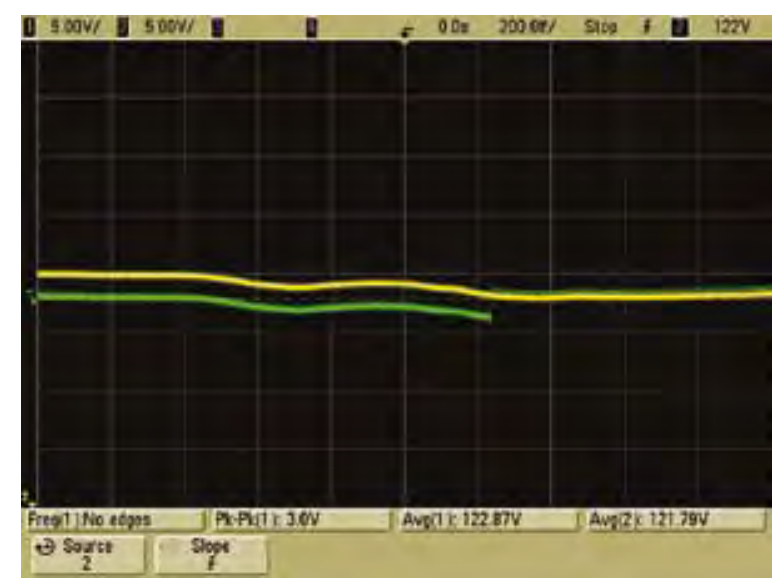

Figura 12: Primera apertura del contactor para asegurar la salida en 122 VCC.

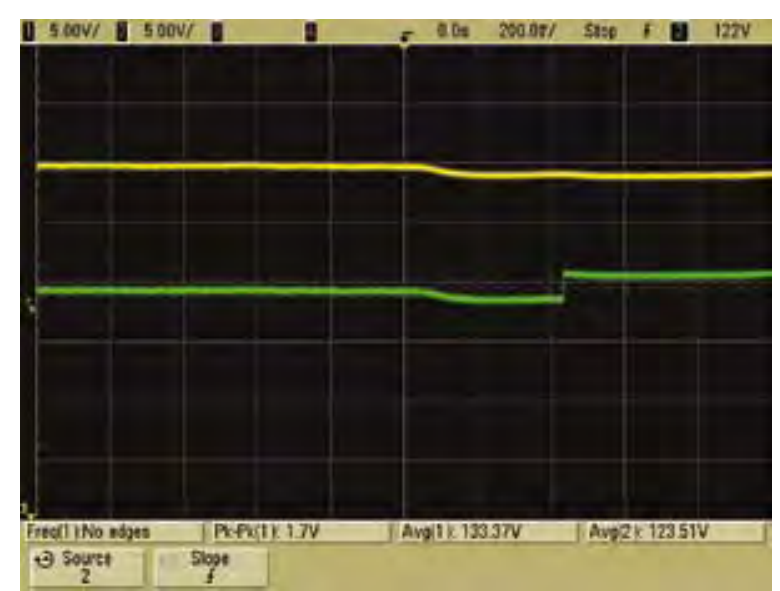

Figura 13: Quinta apertura de contactor para matener la tensión en 122VCC.

\section{CONCLUSIONES}

Con los resultados obtenidos en este trabajo se ha probado que el UDCV satisface los objetivos de regular la tensión en el $125 \mathrm{VCC} \pm 4 \%$. Los resultados para altas potencias no se obtuvieron en el laboratorio, y no son presentados aquí. Sin embargo, la compañía que probó el aquipo final alcanzó resultados satisifactorios para la máxima corriente. Comparados con otras topologías CC$\mathrm{CC}$, el costo/beneficio de esta unidad desarrollada muestra que es la mejor solución, por no requerir grandes inductores, transistores de potencia o gates driver. La implementación de la rutina de control en un microcontrolador también se presenta como una solución atractiva por ser más económica comparada con DSP o FPGA.

\section{AGRADECIMIENTOS}

A la empresa Emerson Networks, por el financiamiento del proyecto que será usado en su línea de producción, y en la mejora y desarrollo de proyectos futuros.

\section{REFERENCIAS}

[1] Tanwir, Zubayer, Islam et al., Eight Switch Buck Boost Regulator Topology for High Efficiency in DC Voltage Regulation, $5^{\text {th }}$ International Conference on Electrical and Computer Engineering, ICECE, Bangladesh, 2008.

[2] S. Saggini, M. Ghioni, and A. Geraci, An Innovative Digital Control Architecture for Low-Voltage, High- Current DC-DC Converters With Tight Voltage Regulation, IEEE Transactions on Power Electronics, Vol. 19, No. 1, Jan. 2004.

[3] L. C. G. Freitas , G. B. Lima , G. A. Melo et al., A Novel Single-Phase HPF Hybrid Rectifier Suitable For Front-End Trolleybus Systems. COBEP, Bonito - MS - Brazil, 2009, 619-626.

[4] F. A. S. Gonçalves, C. A. Canesin, G. A. Melo et al., HPF Boost Interleaved Operating In Discontinuous Conduction Mode For Trolleybus Application, COBEP, Bonito - MS - Brazil, 2009, 648-654.

[5] Muhammadh H. Rashid, Power Electronics Handbook, Academic Press. 2001. pp. 169-224.

[6] N. Moham, T. Undeland, W. Robbins, "Power Electronics Converters, Appplications and design", John Wiley \& Sons, 2003, 161-248.

[7] Ivo Barbi, Eletrônica de Potencia, 3era. Edição. Florianópolis - SC - Brazil, 2000, 101-156. 
[8] J. A. Pomilio, "Eletrônica de Potência", Campinas SP - Brazil, Janeiro, 2002.

[9] ON Semiconductor, "Linear \& Switching Voltage Regulator Handbook” - Rev. 4, Feb-2002.

[10] R. Boylestad, L. Nashelsky, "Dispositivos Eletrônicos e Teoria de Circuitos”, Editora LTC. 6ta Edição, 1999, 554- 557.

[11] Randall Shaffer, "Fundamentals on Power Electronics with Matlab", Charles River Media. Boston USA, 2007, 183-289.

[12] Robert Erickson, Dragan Maksimovic, "Fundamentals of Power Electronics", Kluwer Academic Publishers, Colorado, USA, 2004, 187256.

[13] Demercil S. Oliveira Jr., Luis C. Tomaselli, Estudo de um Conversor CC-CC Buck-Boost, INEP UFSC.

[14] Y. M. Lai, "Power Supplies", In: Muhammadh H. Rashid, Power Electronics Handbook, Academic Press, 2001, 487-506.
[15] A.B.N.T. 240-510-713, Características técnicas sobre fabricação e fornecimento de unidades de diodos de queda (UDQ) tipo 2, para fontes de corrente contínua em telecomunicações, 1977.

[16] A.B.N.T. 240-510-702, Especificaçóes Gerais para fonte de corrente continua Tipo 2 para equipamentos em telecomunicaçóes, 1977.

[17] Norma Técnica Petrobras N-332, "Especificaçóes Carregador de bateria de 125 V" Rev. C. Junho, 1988.

[18] EMERSON NETWORK, Especificaçóes de requisitos para controle de UDQ de sistemas de Retificadores operando em 125Vcc, 2008.

[19] Norman S. Nise, "Control Engineering Systems", Jhon Wiley \& Sons, $3^{\text {rd }}$ Edition, 2004, 499-589.

[20] Marty Brown, “Power Supply Cookbook”, Newes, $2^{\text {nd }}$ Edition, 2001, 21-133.

[21] Alpha \& Omega Semiconductor, Buck Converter Control Cookbook, August 2008.

E-mail: jmontalvan@batlab.ufms.br 